ulcer, $H$. pylori infection was present in 12 children out of 15 (80\%).

Conclusions Peptic ulcer disease remains high in children and the infection with $H$. pylori has a high incidence among this pathology.

\section{P321 DISORDERS OF THE GALLBLADDER FUNCTIONAL STATUS IN THE CHILDREN WITH PANCREATIC REACTIVE CHANGE}

Olesya Horlenko*, Agneta Lenchenko, Olga Pushkarenko, Gabriella Kossey, Adrian Tomey, Viktoria Polyak-Tovt. Uzhhorod National University, Uzhhorod, Ukraine

\subsection{6/archdischild-2019-epa.670}

Introduction Biliary system functional disorders are one of the most common conditions among children and adolescents. The so-called dyskinesias in childhood and adolescence have progression and contribute to the development of organic diseases of the bile-excreting system.

Materials and methods On the basis of the Uzhhorod City Children's Clinical Hospital, 86 children were examined and treated. The main disease in the patients of which was accompanied by pancreas reactive changes. The age of children ranged from 2 to 16 years. Of these, 47 (54.7\%) boys and $39(45.3 \%)$ girls. To evaluate the motor function of the gall bladder and biliary tract, dynamic ultrasound cholecystography (UHC) with dietary stimulation of biliary excretion was used.

Results The vast majority of children $(75(87.2 \%))$ have presentad complaints on vomiting. The data of the objective examination corresponded to the clinical picture of the underlying disease. Paraclinical examination revealed that in 63 $(73.3 \%)$ children urine amylase increased in $41(65.1 \%)$ to $128 \mathrm{U} / \mathrm{l}$, in $12(19 \%)$-to $256 \mathrm{U} / \mathrm{l}$ and in $2(3.2 \%)$ - up to $512 \mathrm{U} / \mathrm{l}$. In $61(70.9 \%)$ children, acetonuria was noted, and in 15 (17.4\%) - hyperglycemia was onset.

According to gall bladder ultrasound examination, its increase was found in $24(27.9 \%)$ patients, deformation was found in $24(27.9 \%)$, sealing of walls - in 12 (13.95\%), biliary precipitate was visualized at $8(9,3 \%)$ children. 15 patients were selected for the possible detection of violations of the gallbladder motor function from the examined group, which were subjected to a detailed ultrasonographic examination - dynamic ultrasound cholecystography (DUCG). At DUCG the phases of gallbladder contraction are registered. With a hyperkinetic type of dysfunction of the gallbladder, the volume of the gall bladder was reduced by more than $65 \%$ at 60-90 minutes after taking a cholecinetic breakfast; with a hypokinetic type was less than $33 \%$.

Summary It was established that in almost half of the children from the selected group $(7(46.7 \%))$ the volume of the gall bladder exceeded the age standards. Functional disorders of the gall bladder were observed in all children with reactive changes in the pancreas: $13(86.7 \%)$ were hyperkinetic, 2 $(13.3 \%)$ under hypotonic type. Volume of the gallbladder in the onset in $46.7 \%$ of children exceeded the age norms.

\section{P322} COMMON THINGS ARE COMMON, EXCEPT WHEN THEY'RE RARE

${ }^{1}$ Jennifer Cox*, ${ }^{1}$ Shoana Quinn, ${ }^{2}$ Melanie Cotter, ${ }^{3}$ Alan Irvine. ${ }^{1}$ Tallaght University Hospital, Dublin, Ireland; ${ }^{2}$ Temple Street Children's University Hospital, Dublin, Ireland; ${ }^{3}$ Our Lady's Children's Hospital Crumlin, Dublin, Ireland

\subsection{6/archdischild-2019-epa.671}

Introduction Blue rubber bleb nevus syndrome (BRBNS) is a rare condition characterised by multiple cutaneous and internal venous malformation. Cutaneous lesions are often small, blue to purple in colour and have a predilection to the palms and soles of the feet. GI lesions are pathognomonic for the disease and most commonly seen in the small bowel. Haemorrhage from venous blebs in the GI tract can cause chronic iron deficiency and anaemia.

Life-long iron supplementation and repeated iron and blood transfusions are required for affected individuals. Treatment options have included sclerotherapy, aggressive surgical removal of GI venous malformations, and more recently, medical treatment with sirolimus.

Case report We present a case of a 9-year-old Irish male with chronic iron deficiency anaemia and multiple vascular lesions.

The patient presented at one year old with a large vascular malformation, likely congenital hemangioma, overlying the occiput which increased in size over time. A trial of propranolol failed to reduce the size of the lesion and it was excised at 18 months old. However, a reoccurrence was noted 5 years after surgery. Other cutaneous stigmata include one vascular lesion on the sole of each foot.

The patient presented again in early childhood with a chronic microcytic anaemia. His anaemia progressed over time, with recurrent drops of haemoglobin requiring frequent blood transfusions. He had a normal haptoglobin level and no evidence of hemosiderin in his urine, suggesting a cause other than haemolysis of the vascular lesion causing the anaemia.

To investigate this unexplained iron deficiency anaemia, he had a colonoscopy at age 6 years. This showed two small haemangiomata in the rectum and descending colon and no evidence of inflammation. Repeat upper and lower endoscopy three years later was normal, with no vascular lesions seen.

The patient subsequently had a capsule endoscopy which demonstrated multiple small bowel angiomata and venous blebs. The pathognomonic cutaneous and small bowel vascular malformations, combined with a recurrent, severe iron deficiency anaemia dependent on blood transfusions are consistent with a diagnosis of BRBNS for this patient. He has recently started on sirolimus, a mTOR inhibitor which has been used for treatment of vascular anomalies in children including BRBNS. Initial response to the treatment looks promising with improvement in haemoglobin one month after starting treatment.

Conclusion We present a rare case of blue rubber bleb nevus syndrome in a 9-year-old boy with multiple venous blebs causing severe, chronic anaemia. 